may witness the spastic, non-congenital / plete paralysis of the whole of the muscles $\mathbf{T}$. varus in the left foot, the right limh of the without any contracture. same individual being affected with com-

Fig. 12.

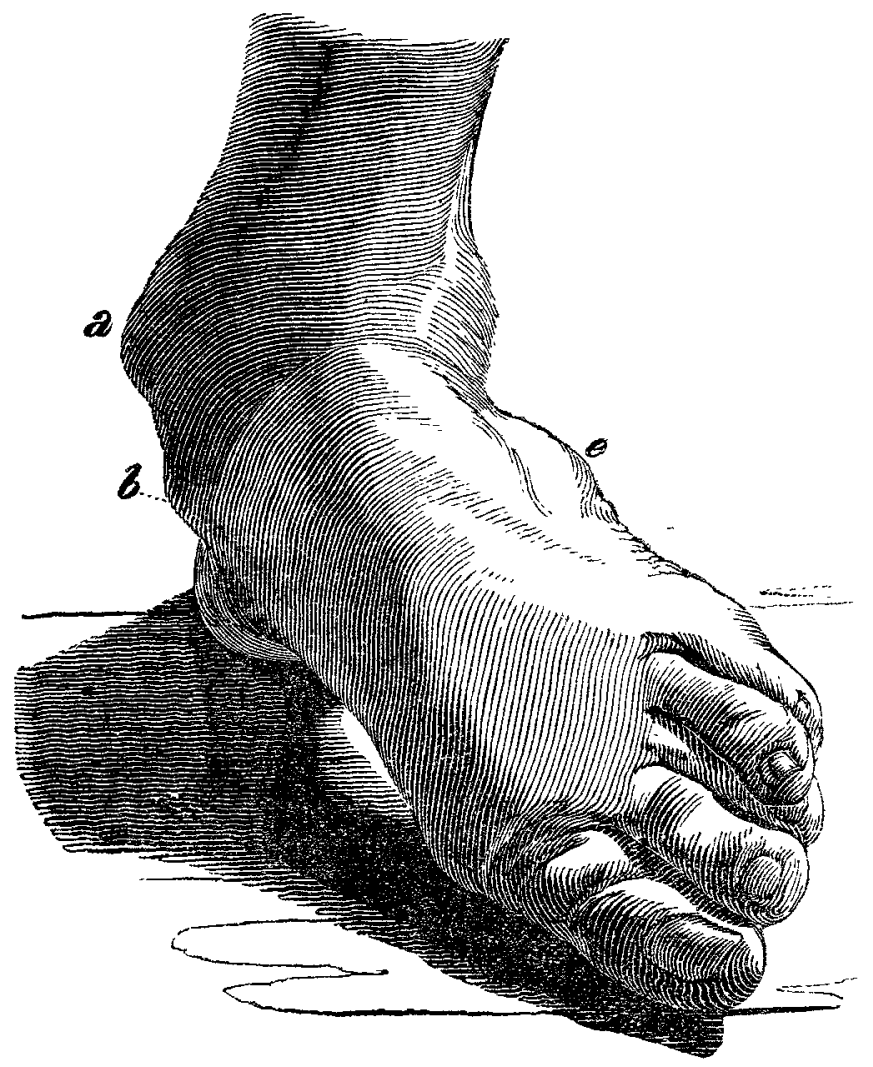

T. calcaneo-valgus, from paralysis of gastrocnemii, and anterior and posterior tibial muscles.

a. The internal malleolus. b. The projecting astragalus.

e. The outer margin of foot raised from the ground.
ON

STAMMERING, ITS CAUSES, VARIETIES, AND TREATMENT.

By James Yearseey, Esq., M.R.C.S.

(Concluded from p. 918.)

MECHANICAL TREATMENT OF STAMMERING.

We see the first germ of mechanical or surgical treatment of impediments of speech in the pebbles, which it was the ancient practice to recommend stammerers to place in the mouth, and which, in the cure of Demosthenes, divided the palm with his dramatic or rhythmical readings. In certain cases this practice is found to be beneficial in regulating the speech. The presence of pebbles, quite large enough to interfere with the function of speech in ordinary persons, exerts a controlling and beneficial effect over the organs of a stammerer. It is the mere presence of the foreign body that seems to influence the disordered speech, the situation in the mouth not appearing to be of mate. rial consequence. The celebrated Madame Leigh, of New York, treated her pupils chiefly by mechanical regulations. Her principal direction was to keep the tip of the tongue firmly pressed against the anterior part of the palate, and in conversation to set off in speech from this point as from a spring. Itard, whose attention, like my own, was first attracted to the subject from observing its frequent association with deafness, bridled the tongue by means of a forked metallic instrument placed under this organ. I have seen cases in which broken teeth aggravated the disorder, and the introduction of artificial teeth led to a cure. From such facts as these I am led to believe that the increased sensibility of the mouth during the latter part of dentition, and the irregularity of the jaws until the full set of teeth is developed, often lay the foundation of impediment of speech.

Cases are on record, and I have myself known instances, of cures having been sud- 
denly and effectually brought about by some accidental injury in the neighbourhood of the organs of voice and speech. For example, laceration of the face or lower-jaw, or burns of the neck. I once had a patient from Edinburgh whose brother, also a stammerer, fell from a height and cut his face. From that moment the stammer left him. Such a re. markable result tempted my patient's friends to try an experiment upon him. They did not, indeed, determine on throwing him from a height, but one night, on ascending the stairs in the dark, he found himself suddenly assailed by a bucket of water dashed full in his face. It knocked him backw ards down the stairs, but no good came of it. The experiment failed.

The same kind of cure has been occasionally effected in other spasmodic affections, particularly chorea and epilepsy, the former of which $I$ have indicated as bearing a near resemblance to stammering. I have also known many instances of the application of a blister upon the larynx or some other part of the neck, for other purposes, entirely control an impediment of speech as long as the blistered surface remained sore. The idea of performing an actual operation in stam. mering is not entirely new, though no at. tempt had heretofore been made to reduce operative measures to any thing like a rule or principle. I recorded a case in my first publication on the subject of stammering, in which Sir Wm. Blizard divided the frænum linguæ with a view to relieve an impediment of speech.

From these circumstances, independently of the operations lately introduced, it is evident that mechanical or surgical measures are occasionally productive of benefit. Of course I look upon the effects of local injuries in removing the disorder as cases of accidental operation. In cases of this kind no mechanical obstruction is removed which might either directly or indirectly have affected the speech. In one case which came under my notice the accident, which caused the cure, wounded a part of the neck removed from the larynx. We cannot explain the effects of such accidents or mechanical causes by fright, az there are no cases knowa in which angthing of this kind has effected a cure, though it has in some instances caused the disorder.

My own opinion is made up that all the foregoing causes of relief, from the simple pebble to the most serious laceration, are to be explained on one similar principle, and this is probably that of their interfering with the morbid action of the nerves supplying the parts concerned in speech. It seems as though a cause which would interfere with speech under natural circumstances, will sometimes check the irregular action which constitutes stammering.

The medical treatment of stammering is of a simple kind, being that which is adapted to restore the strength in cases where the general health suffers. Stammering is an affection which sympathises in an extraordinary degree with the state of the general health, being always least troublesome at those limes when the individual affected is in the most healthy condition, and'vice versat. The most marked examples of the influence of medical treatment occur in cases of stanimering complicated with chorea. The cure of the choreal disease invariably exerts a good effect on the vocal impediment.

\section{CLASSIFICATION OF STAMMERING.}

The declamatory processes so long used by the teachers of elocution have been, in an eminent degree, empirical. They have known or admitted no division of the disorder into different kinds or modifications, but have uniformly treated it as one affection, and as such requiring one remedy. But how stands the fact? I have already shown that great diversity exists in the characteristics or symptoms of different cases, and these not of an accidental but of a constant kind; sufficient, as I conceive, to warrant a division of the disorder into several forms, each depending on lesion of some special part of the extensive apparatus of respiration, voice, and articulation. There are cases in which disordered respiration is the chief cause of the impediment, voice and speech being produced only as long as the breath can be drawn regularly. There are others in which the function of the laryng is entirely suspended at intervals, the organs of articulation being unaffected by the spasmodic action. In another class the breath is free, and a full sound issues from the largnx, but some uncontrollable force renders the organs of articulation unable to perform their task. Reason would point to a difference of treatment for these op posite forms of disorder, and my experience confirms the utility and suc. cess of following her dictates.

The operations performed by Dieffenbach, of making a section of the tongue, or removing a portion of it, and those of Amussat and Phillips, of dividing certain of the lingual muscles, were also of an empirical nature. They were performed indiscrimiately in all cases of stammering, no rules being attempted to be laid down for the application of these severe measures to individual cases. It was sufficient for the patient to express his readiness to run the risk of the experiment. I must admit that the same remarks apply to my own earlier operations. Judging from the immediate effects, I concluded that the tonsils should be removed in all cases in which these glands were diseased and enlarged, and if this were not the case, or the operation when performed did not relieve the impediment, I had recourse to excision of the uvula in all cases irrespectively. It is a source of regret to me that more discrimination did not mark the outset of my 
surgical treatment; still I may congratulate myself that, unlike the operations of the continental surgeons, no harm ever resulted from mine, although, for reasons which it is unnecessary to advert to here, a surgeon who has arrived at eminence in his profession chose to denominate the operations I proposed " horrible mutilations of the palate, and unwarrantable incisions."

But to return. Further experience has convinced me of the error of these opinions, and has shown that the greatest amount of benefit can be obtained by diversifying the operative measures, and conjoining them with other means according to the variety of the impediment.

The following are the different forms of stammering, which, I believe, can be satisfactorily made out :-

1. Respiratory Stammer -In which respiration is at fault, the patient appearing to be unable to bring the air up from the lungs to act on the larynx; the chest frequently becoming in the attempt to speak as fixed and immoreable as it is in tetanus.

2. Laryngeal Stammer-In which the impediment occurs in the larynx, no sound being produced, or merely a hissing by the passage of air through the vocal organ; in some cases the aperture of the glottis is closed, or nearly so; in all there is violent muscular action about the larynx which can be seen externally.

3. Guttural Stammer - In which the obstruction is distinctly referred to the top of the throat and soft palate.

4. Lingual Stammer-The difficulty consisting in the inability to manage or control the action of the tongue, this organ being sometimes fixed immoreably, in others moving about convulsively in the mouth, or thrust out between the teeth.

5. Labial Stammer-Here the impediment appears seated in thelips, which are in general firmly compressed together.

6. Facial Stammer - In which not only the lips, but the muscles of the face generally are convulsively affected.

7. Choreal Stammer-This sometimes occurs in combination with chorea; but more common the attempt to speak brings on choreal movements of the face, head, neck, arms, and sometimes the whole body. In this variety the irregular motions take place in all the parts concerned in speech, from the chest to the lips inclusive.

8. Mixed Stammer-Here the characteristics of the various kinds of stammer just enumerated meet in different combinations; in some cases one variety appearing promiuent, in others the symptoms being indiscri. ininately mixed.

\section{TREATMENT OF STAMMER.}

1. Respiratory Stammer-This is the most difficult to treat of all the varieties. Operative measures are here, I believe, entirely without efficacy. The affection is not confined to one part, as in most of the other kinds, but the whole function of the respiratory motor apparatus seems thrown into dis. order immediately the attempt is made to speak. The chief indications are to restore the inspirations and expirations" from their irregular and spasmodic action to their natural rhythm, to bring the respiration into harmonious co-operation with the organs of voice and speech, and in certain cases to strengthen the lungs by appropriate exercises.

In cases of respiratory stammer the chest will almost always be found of small capacity, and the breath is what is termed short. When the patient is young, recourse may be had to those gymnastic exercises which tend to expand the chest and strengthen the upper part of the body. Rowing, the use of the dumb-bells. and other exercises of the same kind, are therefore useful. I consider the practice of singing of much service in giving sustaining power to the breath. Playing on wind instruments, such as the fute and clarionet, have the same effect, and should be recommended. Music, also, on another account, is desirable : it tends to set up in the patient's mind the rhythmical precision in which he is, or rather bis vocal organs are, deficient. Reading aloud, especially of dramatic poetry, should be practised two or three hours a-day, with a view to strengthen the chest voice. Demosthenes acquired strength by practising on the sea shore to the noise and rhythm of the waves. Not only should loudness and power of voice be attempted, but a certain amount of rhythm should be introduced in the respiratory efforts, on which all voice primarily depends, and which, in respiratory stammering, have the chief share in producing the impediment. In natural respiration breathing goes on in a rhythmical manner, expiration and inspiration follow each other at regular intervals. This regularity is preserved to a certain degree in natural speech; only the respirations are taken quickly, and the expirations are prolonged; but in the speech of a stammerer with the variety of impediment we are considering, the respiratory rhythm is almost entirely lost during speaking. The individual takes long and short inspirations and expirations with such irregularity that he constantly appears gasping for breath. In some instances a deep inspiration is only now and then procured, and when it is obtained the words are poured out in a torrent as long as the supply of breath can be kept up. These irregularities may all be cbecked by a plan which causes the inspirations and expirations to follow each other in the same intervals of time. The points and stops of reading and speaking should be made at shorter intervals than usual, and these intervals should be as strictly regular as possible. A full 
breath should be taken at every stop. A stammerer can no more talk without a supply of breath than a steam engine can move without its due complement of fuel.

This idea of respiratory scanning should also be introduced into ordinary speech, and should, if possible, be carried out with such exactitude that the breath of a stammerer while speaking should be as regular and unintermittent as the breathing of a healthy person during sound sleep. It is astonishing how rapidly the practice of this rule will benefit a case of pure respiratory stammer. ing.

It must, in addition, be insisted on with as much earnestness as anything which has been said abore, that a stammerer of this class should never attempt to speak during the act of inspiration. Some bave a vicious habit of speaking only while the breath is being drawn in, and seem in a continual effort to swallow their words; whereas, in natural speaking, and in all attempts to correct faulty modes of speech, the words should float out on the top of the stream of breath.

2. Laryngeal Stammer.-This variety of stammer, though not, like the former, entirely removed from the influence of operative treatment, is less amenable to its employment than the varieties which follow. Something may yet be done by surgical means which, in many cases, will prove of benefit: for instance, burning a moxa over the hyoid bone, externally, so as to produce a small eschar, applying a blister or producing decided irritation by means of tartaremetic ointment. This treatment will frequently control the violent spasmodic action of the larynx, which sometimes, in these cases, rises up full two inches from the nata. ral position; and there is little doubt that this visible convulsion is attended by excessive action of the internal muscles of the vocal organ.

Correctional training is of immense importance in laryngeal cases. One of the peculiarities of the affection is that the stammering frequently occurs with most insupe. rable force at the open or vowel sounds. These sounds can, however, even in the most desperate cases, be readily emitted if a singing, chaunting, or, in other words, a rhythmical method be adopted. During the process of cure everything should be spoker as though it were written in rhyme, the rhythm being gradually dropped as an easy mode of utterance is obtained, till at length it may become almost imperceptible. The facility of applying the rhythm is increased by the irritant treatment of the laryngeal region externally. The application of rhythm to the voice may be still more facilitated by marking the time with the foot, the hand, or the head, care being taken that the movement be synchronous with the waves of sound issuing from the larynx. Those who have a correct idea of musical time, or rhythm, do not require any such action to regulate the speech. Such persons can set up the rhythm in the mind and carry it out in their conversation.

This, then, is a plain outline of one of the most boasted and oracular mysticisms of the elocutionists. This class of persons has been accustomed to extort enormous fees for its communication, and to occupy weeks or months in imparting that which any intelligent person may not only comprehend but apply in a very few minutes.

3. Guttural Stammer. - This is the variety of stammering in which, beyond all others, I have proved operative measures to be of great immediate and permanent benefit. It is to this variety which, after nearly three years most careful observation of the disorder, with unprecedented opportunities of investigation, I am disposed to limit the operation I was the first to propose, namely, excision of the urula. I trust it will not be considered out of place again to advert to the circumstances which first led me to the performance of this operation and excision of enlarged tonsils as a means of cure for stammering. While engaged in the treatment of deafness, I removed several diseased tonsils in cases where the malady appeared to be dependent on this morbid condition of the throat, with which stammering co-existed. This was done solely with a view to restore the hearing, and was quite accidental as regarded the vocal affection. However, I found that not only was the hearing improved or restored, but the speech at the same time lost its impediment.

Thus a new and interesting field of research was opened to me. At first the favourable results of my operations led me to believe that a specific had been discovered for this distressing affection; but with greater experience it soon became evident that certain operations were adapted only to certain varieties of impediment, and that in many cases, when applied with the necessary discrimination, they were sufficient of themselves to perform a cure, and that they always present a favourable basis on which to proceed with educational training and correction.

It is not to be wondered at, that I should be assailed and misrepresented by those whose professional calling was likely to be interfered with by my innovations of treatment. It has suited the purposes of these persons to represent my treatment as purely operative, and as consisting of " borrible mutilations." It is scarcely necessary for me to declare that I have never removed tonsils except when these glands are in a diseased state, for when they are not in a discased state they cannot be remored. With respect to the uvula, $I$ do recommend its removal 
irrespective of its own condition, in the guttural variety of stammering, but in no other instance would $I$ interfere with this body, unless, indeed, its diseased condition rendered its removal a peremptory duty.

Much has been said about the theoretical evils which may result from the loss of the uvula, or the removal of diseased tonsils, but no one has ever shown a single case in which either the absence of an uvula, as a natural condition from birth, its removal by disease, or its excision, has produced any of the effects which have been laid to the charge of this operation. Surgery would for ever have remained an insignificant art had it been the custom of its professors to hesitate in effecting a positive good from apprehension of a merely imaginary evil. I can readily con. ceive that many persons may object to the excision of a healthy uvula, though I am convinced of the futility of the objection, but $\boldsymbol{I}$ am utterly at a loss to conceive how any one can object, in the case of enlarged tonsils in cases of stammering, to remove a positive and constant irritation from the parts affected in this disorder.

Within the first few days after the guttural operation, whether the uvula or the tonsils have been removed, there is frequently no sign of the previous impediment. To prevent its return $I$ recommend the assiduous observance of rhythm in the manner already explained. When the operation appears from the first only pallative, rhythm should be practised unremittingly from the moment of the operation until the total disappearance of the impediment, and even for some time afterwards.

4. Lingual Stammer.-It is to this variety of stammering that the operations of Dieffenbach and A mussat, on the tongue and lingual muscles, would be applicable, were it not that their severity renders their performance altogether inexcusable. Both these operations have proved fatal. even in the most experienced and skilful hands, and as stammering is considered more in the nature of a personal defect than a disease, and as it never endangers life nor appears to shorten it by its continuance, all operations attended with risk are unjustifiable.

There is nothing in the nature of stammer. ing, nor in the experience gained relative to its treatment by operation, which tends to prove that the amount of benefit is in a ratio with the severity of the operation performed. There is no doubt that pebbles in the mouth, the lingual posturising of Madame Leigh, the metallic fork of Itard, and snipping the franum lingux, have all occasionally produced cures, as well as the most severe operations. The true object to be aimed at seems to be the setting up of an irritation or interference with the movements of the tongue, and this, instead of increasing the disordered action already existing, tends to quell or neutralise the primary disturbance. I do not illustrate the point thus to build up any theory of my own, but merely to de. scribe what actually occurs. The operation I perform in lieu of the above is to introduce a seton, of several threads of silk, by means of a curved needle deeply into the frænum of the tongue so as to include a portion of its muscular substance. The silk is then tied in a knot, and suffered to remain until it sloughs away. The operation and the presence of the silk cause a soreness in the mouth which appears to have a most remarkable influence in controlling the irregular action of the tongue, and the stammerer, whose chief difficulty lay in the lingual letters $d, t, r, 1, \& c$. , suddenly finds himself relieved of his impediment. In a short time the silk becomes thickly encrusted with a deposition of tartar, which probably assists in the good effects of the operation.

In addition to the operation, the patient should be schooled into the employment of rhythm, for there is scarcely a case which will not progress more rapidly towards a perfect cure by its potent agency. In obstinate cases this frænum operation may be repeated with advantage.

5. Labial Stammer.-In this very frequent variety of stammering $I$ perform a modification of the measure recommended in lingual cases; $I$ introduce a seton of the same kind into the inferior frænum labia, and sometimes also into the frænum of the upper lip. It is interesting to observe how instantaneously the stammer sometimes takes its departure from the lips upon this operation being performed. Words commencing with $b, p$, or $m$, are immediately articulated with ease. Sometimes the difficulty is at once and for ever surmounted; sometimes the operation must be repeated. Rhythm, as in all the other varieties, must be again brought into play; for it is rare, indeed, to see a case of stammer confined to the lips; as in lingual or guttural stammer it will be found associated with more or less of respiratory stammer. The practice recommended by $\mathbf{D r}$. Arnott is of great utility both in lingual and labial cases, namely, to produce a slight sound, as of $e$ French, in the larynx before the articulatory sound is produced in the mouth. 'The vowel sound may be so closely uttered with the consonant as to be undistingnishable, edark, ebright, emorning. If we examine into what happens during the production of speech, we shall find that this is only doing, in slight excess, that which always takes place in natural speaking. The rowel or laryngeal sound must be produced before the lingual, labial, or guttural modulation can be performed, though in ordinary speech the one follows the other so rapidly that the first is not perceived.

$\Lambda$ nother adjuvant or remedial measure of considerable power is the practice of speak- 
ing aloud alliterative sentences, or words indiscriminately selected, beginning with the same letter, such as Bath, Bristol, Bridgwater, Brighton, Buxton, \&c.; or Paul Potter, Peter Piper, and so forth. This kind of practice is well adapted to stammerers who are caught at a particular letter.

6. Facial and Choreal Stammer are generally found associated with severe respiratory stammer. It is the variety of impediment which, beyond all others, requires medical treatment. The usual remedies for chorea, such as purgatives and tonics, pure air, a generous diet, must be resorted to on account of the general bealth. With reference to the roice and speech especially, all the educational means adverted to should be adopted. The chief indication is to give to the organs of respiration, voice, and speech, a regular action as regards each, individually considered; and, in addition to this, we shall gain but little if we do not promote a regular and harmonious combination of action between all the organs collectively.

7. Lastly, in the Mixed Variety the different curative agents, operations, rhythm, and medical treatment, must be brought to bear singly or combinedly, according as one or another variety has the greatest prominence in the cases to be treated:-the aim in all cases being to combat the particular kind of impediment, to give general strength, to inspire mental confidence, and to impart power, precision, and regularity, to the functions of respiration, voice, and articulation.

Saville-row, Nov. 1813.

\section{RESEARCHES IN EMBRYOLOGY.}

COFCLUSION OF MR. JONES'S REPORT ON THE DEVLLOPNENT OF THE OVUM. ANNOCNCEMENTS OF M. SEIERES ON THE ALLANTOIS, ETC., IN THE HUMAN OVUM.

$I_{N}$ the last volume of THE LANCer we promised to notice, at a convenient opportunity, the researches of M. SERres into the presence of the allantoid membrane in the human ovum during the earlier period of its development, and an occasion now occurs to redeem this promise. Completing our analysis of Mr. Wharton Jones's Report (see last No., p. 258), to whose researches those of M. Serres are intimately allied, we shall conclude the subject with the latter.

Development of the Embryo.-We bave traced the ovum into the uterus and remarked on the first changes of the blastodermic vesicle, the production of the area pellucida, area opaca, \&c. Coste, and after him Bischoff, showed a perfect analogy to exist in the development of the embryo, not only among mammifera, but between them and birds. As has been seen (ante, pp. 261-2), "after the area germinativa comes to present a central pellucid and a peripheral opake part, and passes from the round to the pearshape, a bright streak appears on the long axis of the pellucid part." Close around this streak, but especially on either side of it, formative opake matter collects, principally in the animal (serous) layer. The bright streak mentioned is a groove in that layer; at that end of it corresponding with the broad end of the pear-shaped iarea, its two edges " pass into each other by a snall arch, whilst at the other end they unite in a point. The former is the head, the latter the tail end of the embryo."

The regetative (mucous) layer is almost uniformly opake in the whole extent of the area germinativa; it also presents a faint clear streak, but this is supposed to be merely an impression made by the groove of the animal layer, for, at the groove, the two layers intimately adhere.

Formation of the Spinal Canal and Viscerat Carity.-The area pellucida having become fiddle-shaped, the opake formative matter in the animal layer, where it immediately constitutes the sides of the groove, rises up in the form of dorsal plates, which meeting in the middle, over the groove, convert it into the canal in which the central organs of the nervous system are formed. "Subsequently, the circumferential part of the same forma. tive matter becoming thickened, and bending downwards and inwards, constitutes "visceral plates.' The area pellucida is now reduced to a crescentic patch around the head of the embryo.

6. From this it is seen that the first develop. ment of the embryo of the mammifera is aualogous to that of the embryo of the bird. Its further development is so likewise. The 'dorsal plates' having closed in, the formation of the nervous centres commenced, and the head end of the dorsal canal become dilated, the embryo bends forward on itself, and an eleration of its anterior, and, in a less degree, of its posterior end, takes place from and above the plane of the blastodermic resicle, so that the embryo is, as it were, constricted off from it," The margins of the "visceral plates" all round approach each other, and inclose the "visceral cavity;" and gradually these plates also separate off from the blastodermic vesicle behind. The vegetative follows the direction given by the animal layer in this process; and above and below it is drawn within the visceral tube now in progress of formation; but from the points of constriction, or places where it so enters the visceral tube, the vegetative layer is continued over the head and tail ends of the embryo, the coverings it thus forms being termed the head and tail involucra.

Formation of the Amnion.-In the formation of the above involucra, the animal (a: 